\title{
Bulk and Interfacial Wetting Behavior of Binary Mixtures Induced by Associating between Unlike-Pair Molecules
}

\author{
Chia-Ming Chen, Ming-Chih Yeh, and Li-Jen Chen* \\ Department of Chemical Engineering, National Taiwan University, Taipei, Taiwan 10617, Republic of China
}

Received: November 2, 2005; In Final Form: January 2, 2006

\begin{abstract}
The statistical associating fluid theory of Wertheim is applied to describe binary mixtures with associating between unlike-pair molecules. The phase behavior of this binary mixture would fall into five different types (I, II, III, V, and VI) of the classification scheme of van Konynenburg and Scott by varying the associating strength and the energy parameters. Both interfacial wetting behavior and wetting transitions are carefully examined in all the vapor-liquid-liquid $(\gamma-\beta-\alpha)$ three-phase-coexisting regions of the binary mixtures. The global wetting behavior and wetting transitions are delineated by scanning the parameter space. In certain regions, the middle $\beta$ phase exhibits interfacial phase transitions sequentially, nonwetting $\rightarrow$ partial-wetting $\rightarrow$ nonwetting, at the interface separating lower $\alpha$ and upper $\gamma$ phases along with increasing temperature.
\end{abstract}

\section{Introduction}

Consider a system of three phases $\alpha, \beta$, and $\gamma$ in equilibrium, as schematically shown in Figure 1. The densities of these three phases are in the order $\rho_{\gamma}<\rho_{\beta}<\rho_{\alpha}$. When a small amount of the middle $\beta$ phase is put onto the interface between the upper $\gamma$ phase and the lower $\alpha$ phase, three different geometrical configurations of the $\beta$ phase are possible. They can be distinguished by the contact angle $\theta$, defined in Figure 2, spanned by the $\alpha-\beta$ and the $\beta-\gamma$ interfaces for the droplet of the middle $\beta$ phase. First, if the contact angle is zero, the droplet spreads across the interface to form a macroscopically thick film. The middle $\beta$ phase completely wets the $\alpha-\gamma$ interface. Explicitly, the middle $\beta$ phase exhibits a complete-wetting behavior at the $\alpha-\gamma$ interface, as schematically shown in Figure $3 \mathrm{a}$. Second, if the contact angle is between 0 and $180^{\circ}$, the middle $\beta$ phase remains a droplet suspended at the $\alpha-\gamma$ interface, as shown in Figure $3 \mathrm{~b}$. That is, the middle $\beta$ phase exhibits a partial-wetting behavior at the $\alpha-\gamma$ interface. Third, if the contact angle is $180^{\circ}$, the middle $\beta$ phase dewets the $\alpha-\gamma$ interface and exhibits nonwetting behavior, as illustrated in Figure 3c. In this case, if a substantial amount of $\beta$ phase is added to the system, the $\beta$ phase will not form a droplet shape suspended at the $\alpha-\gamma$ interface due to the gravitational forces. A small amount of the $\alpha$ phase would intrude the interface separating the $\gamma$ and $\beta$ phases to form a complete-wetting layer, as illustrated in Figure $3 \mathrm{c}^{*}$.

A wetting transition is an interfacial phase transition from partial-wetting to complete-wetting (or nonwetting), and vice versa. The occurrence of a wetting transition had been successfully predicted by the phenomenological arguments of Cahn ${ }^{1}$ and by the density functional theory approach of Ebner and Saam. ${ }^{2}$ Consider a three-phase-coexisting system with a partialwetting middle phase, when the system is far from its critical end point. As the system approaches a critical end point, the middle phase should completely spread across (or dewet) the interface separating the other two phases. That is, as a multiphase-coexisting system is brought close to a critical end

* To whom correspondence should be addressed. Electronic mail: ljchen@ntu.edu.tw.

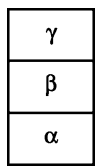

Figure 1. Schematic illustration of a three-fluid-phase $\alpha, \beta$, and $\gamma$ coexisting system in equilibrium.

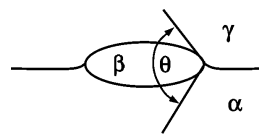

Figure 2. Definition of contact angle $\theta$ in a three-fluid-phase-coexisting system.

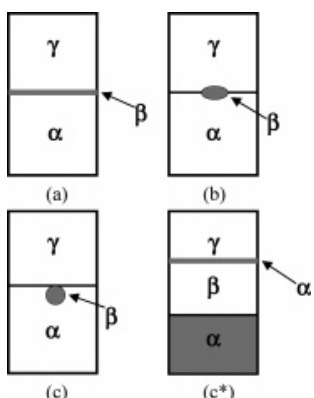

Figure 3. Wetting behavior at a fluid-fluid interface: (a) a completewetting $\beta$ phase at the $\alpha-\gamma$ interface; (b) a partial-wetting $\beta$ phase at the $\alpha-\gamma$ interface; (c) a nonwetting $\beta$ phase at the $\alpha-\gamma$ interface; and (c*) a complete-wetting $\alpha$ phase at the $\gamma-\beta$ interface.

point, these theoretical works predict that an interfacial phase transition must occur from a partial-wetting behavior to a complete-wetting (or nonwetting) behavior. This is renowned as critical point wetting theory. ${ }^{3}$

Such a transition had been experimentally observed in a vapor-liquid-liquid system of cyclohexane and methanol, ${ }^{4}$ as well as in some other binary,,$^{5-7}$ ternary, ${ }^{8,9}$ and quaternary systems. ${ }^{10}$ In addition, it was found that the wetting transition of a water + oil + amphiphile system in the three-liquid-phasecoexisting region occurs as the system is driven to either one of its critical end points by varying the temperature, ${ }^{11-16}$ by varying the amphiphilicity, ${ }^{11,17}$ by tuning the salinity, ${ }^{18,19}$ and 
by varying the oil chain length. ${ }^{20,21}$ In fact, there are a tremendous number of binary mixtures accessible to experiments. However, only a small subset of possible binary mixtures has been experimentally observed to study their interfacial wetting behavior. More precisely, most of the experimental studies of binary mixtures in the literature ${ }^{4-7,22-27}$ belong to the type II or type III mixture groups of van Konynenburg and Scott. ${ }^{28}$ Recently, the wetting behavior for type VI mixtures of van Konynenburg and $\mathrm{Scott}^{28}$ had been explored experimentally.

Some theoretical works on wetting behaviors and wetting transitions employ lattice models. Gompper and Schick ${ }^{29}$ made an extensive review on this approach. An alternative approach is the usage of density functional theory. Vast investigations on the structure of interfaces, density profiles, interfacial tensions, and wetting behaviors of binary mixtures by density functional theory have been performed at vapor-liquid, liquidliquid, solid-gas, and solid-liquid interfaces. ${ }^{30-38}$ These studies are mainly restricted to type II and type III mixtures. Very few studies have examined the wetting behavior as well as wetting transitions in type VI mixtures. ${ }^{39}$

Recently, a simple off-lattice model ${ }^{40,41}$ has been proposed to describe the phase and interfacial behaviors of ternary water + oil + amphiphile mixtures successfully. The association between water and amphiphiles is treated by Wertheim's theory $^{42}$ for associating fluids. The resulting ternary phase diagrams resemble experimental results both qualitatively and quantitatively. The dramatic lowering of the interfacial tensions between the oil-rich phase and the aqueous phase can also be well described. In addition, reentrant wetting and dewetting behaviors have been found in a binary mixture with one selfassociating component at vapor-liquid interfaces. ${ }^{43}$ In this study, this simple model ${ }^{39-41}$ is applied to explore the wetting behaviors at the vapor-liquid interface of the binary mixtures with association between unlike-pair molecules in the region of vapor-liquid-liquid three-phase-coexisting states. By varying the associating strength and energy parameters, five different types, I, II, III, V, and VI, of mixtures are found in this model system. The wetting behavior and wetting transitions in these mixtures are carefully explored in this study. The variety of binary mixtures is systematically classified by the different features of their phase behaviors according to van Konynenburg and Scott. ${ }^{28}$ Figure 4 shows the five different phase diagrams of type I, II, III, V, and VI mixtures accessible in this study.

This manuscript is organized as follows. In the next section, the relationship between interfacial tensions and wetting behaviors is generally described. The free energy model is introduced in section 3. Then, the model is applied to evaluate phase diagrams of vapor-liquid-liquid equilibrium in section 4. The density functional theory is used to calculate the density profiles and interfacial tensions, and the detail of the calculation procedure is given in section 5. The results for the phase diagrams, density profiles, interfacial tensions, and wetting behavior of model mixtures for various energy parameters are presented in section 6. Finally, we make some concluding remarks in section 7 .

\section{Interfacial Tensions and Wetting Behaviors}

For a three-phase, $\alpha, \beta$, and $\gamma$, coexisting system, the wetting behavior is related to the interfacial tensions, $\sigma_{\alpha \beta}, \sigma_{\alpha \gamma}$, and $\sigma_{\beta \gamma}$ corresponding to the $\alpha-\beta, \alpha-\gamma$, and $\beta-\gamma$ interfaces of coexisting phases, and can be classified into three cases.

$$
\sigma_{\alpha \gamma} \geq \sigma_{\alpha \beta}+\sigma_{\beta \gamma}
$$

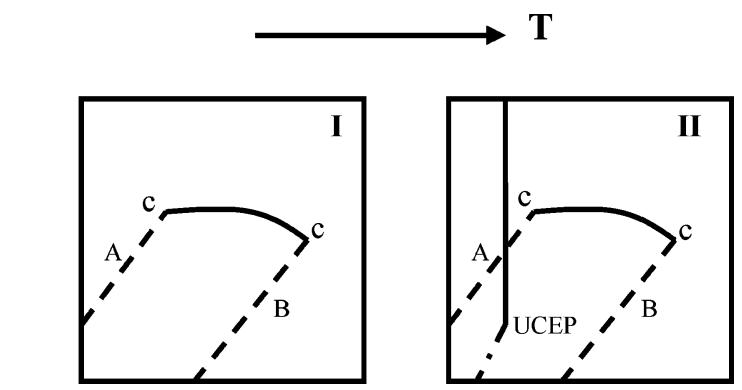

$\mathbf{P}$

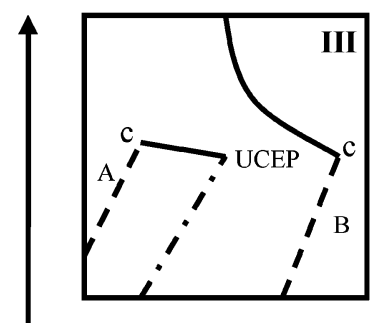

(c)

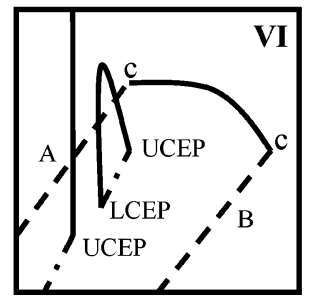

(e) (b)

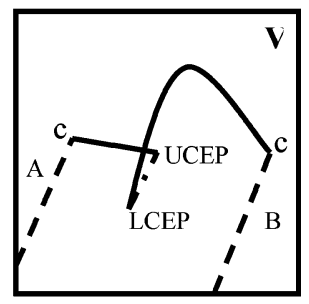

(d)
Figure 4. Classification scheme of phase behaviors for binary mixtures according to van Konynenburg and Scott: ${ }^{28}$ (a) type I mixture; (b) type II mixture; (c) type III mixture; (d) type V mixture; and (e) type VI mixture. The critical loci (solid curves), the critical points of pure components $(\mathrm{C})$, the vapor pressure curves of pure components $\mathrm{A}$ and B (dashed curves), the three-phase vapor-liquid-liquid coexisting (dashed-dotted curves), the upper critical end point (UCEP), and the lower critical end point (LCEP) are illustrated.

The relation between the three interfacial tensions satisfies Antonow's rule ${ }^{44}$ when the equals sign is held. Under this condition, the $\alpha-\gamma$ interface is thermodynamically unstable. Therefore, the $\beta$ phase completely spreads across the interface between the other two phases $\alpha$ and $\gamma$ to minimize the total system energy, as shown in Figure 3a. Explicitly, the middle $\beta$ phase exhibits a complete-wetting behavior at the $\alpha-\gamma$ interface. The contact angle $\theta$ vanishes.

$$
\sigma_{\alpha \beta}-\sigma_{\beta \gamma}<\sigma_{\alpha \gamma}<\sigma_{\alpha \beta}+\sigma_{\beta \gamma}
$$

The relation between the three interfacial tensions satisfies Neumann's inequality, ${ }^{45}$ and all three interfaces are thermodynamically stable. As a result, a small amount of the middle $\beta$ phase forms a lens floating at the $\alpha-\gamma$ interface, as shown in Figure 3b, and exhibits a partial-wetting behavior at the $\alpha-\gamma$ interface. Accordingly, the contact angle for this lens is between 0 and $180^{\circ}$

$$
\sigma_{\beta \gamma} \geq \sigma_{\alpha \beta}+\sigma_{\alpha \gamma}
$$

This relation infers that the $\beta-\gamma$ interface is thermodynamically unstable. Widom ${ }^{46}$ had argued that the free energy of the $\beta-\gamma$ interface can be lowered by an intruding thin layer of the $\alpha$ phase separating the other two phases, $\beta$ and $\gamma$. Explicitly, the surface forces defeat the gravity to budge a small amount 
of the lower $\alpha$ phase up to the $\beta-\gamma$ interface to form a thin intruding layer separating these two phases, as shown in Figure $3 \mathrm{c}^{*}$. That is, the $\alpha$ phase wets the interface separating the $\beta$ and $\gamma$ phases and the relation between the three interfacial tensions satisfies Antonow's rule, $\sigma_{\beta \gamma}=\sigma_{\alpha \beta}+\sigma_{\alpha \gamma}{ }^{44}$ If only a small amount of the $\beta$ phase is present in the system, the middle $\beta$ phase remains in a lenticular shape and exhibits a nonwetting behavior at the $\alpha-\gamma$ interface $\left(\theta=180^{\circ}\right)$, as shown in Figure $3 \mathrm{c}$. On the other hand, the whole $\alpha$ phase, not only a thin film anymore, would separate the $\beta$ and $\gamma$ phases under the condition of no gravity.

\section{Free Energy Model}

The binary associating systems are modeled as mixtures of equal-sized hard-sphere particles A and B. Each molecule is assumed to have an active site that allows association only between unlike-pair molecules to mimic the hydrogen bonding between $\mathrm{A}$ and $\mathrm{B}$ molecules.

On the basis of thermodynamic perturbation theory, the total Helmholtz free energy density of the mixture $f_{\mathrm{T}}$ can be written as $^{39-42}$

$$
f_{\mathrm{T}}=f_{\mathrm{R}}+f_{\mathrm{A}}+f_{\mathrm{M}}
$$

where $f_{\mathrm{R}}$ is the Helmholtz free energy density of the hard-sphere reference term, $f_{\mathrm{A}}$ is the contribution due to the association between unlike molecules, and $f_{\mathrm{M}}$ is the van der Waals meanfield term due to the isotropic long-range attraction forces.

The Carnahan-Starling expression ${ }^{47}$ for hard-sphere mixtures is used to describe the repulsive reference contribution. For all molecules with the equal-sized hard-sphere diameter $d$ in the mixture, the Helmholtz free energy density of the hard-sphere reference fluid is then given by

$$
f_{\mathrm{R}}=\mathrm{k} T \sum_{i} \rho_{i}\left[\ln \rho_{i}-1+\frac{4 \eta-3 \eta^{2}}{(1-\eta)^{2}}\right] \quad(i=\mathrm{A}, \mathrm{B})
$$

where $k$ is the Boltzmann constant, $T$ is the absolute temperature, $\rho_{i}$ is the total number density of substance $i$, and the total packing fraction $\eta$ is defined by

$$
\eta=\left(\pi d^{3} / 6\right) \sum_{i} \rho_{i}
$$

Wertheim's theory ${ }^{42}$ for associating fluids is applied to describe the temperature dependence of the free energy change due to hydrogen bonding. In this study, associations are limited to only occur between unlike-pair molecules A and B. For each molecule with only one attractive bonding site, the association contribution to the free energy can be expressed as ${ }^{42,48}$

$$
f_{\mathrm{A}}=k T \sum_{i} \rho_{i}\left[\ln \chi_{i}-\frac{\chi_{i}}{2}+\frac{1}{2}\right]
$$

where $\chi_{i}$ is the fraction of molecules of type $i(i=\mathrm{A}, \mathrm{B})$ without hydrogen-bonding with another molecule of the other type. The latter quantities are obtained by solving the following mass action equations simultaneously.

$$
\begin{aligned}
& \chi_{\mathrm{A}}=\frac{1}{1+\rho_{\mathrm{B}} \Delta_{\mathrm{AB}} \chi_{\mathrm{B}}} \\
& \chi_{\mathrm{B}}=\frac{1}{1+\rho_{\mathrm{A}} \Delta_{\mathrm{AB}} \chi_{\mathrm{A}}}
\end{aligned}
$$

The quantity $\Delta_{\mathrm{AB}}$ characterizes the strength of the association between molecules $\mathrm{A}$ and $\mathrm{B}$ and is approximated by ${ }^{39-42}$

$$
\Delta_{\mathrm{AB}}=4 \pi g_{\mathrm{HS}}(d) K_{\mathrm{AB}} F_{\mathrm{AB}}
$$

where the parameter $K_{\mathrm{AB}}$ is the bonding volume and $g_{\mathrm{HS}}(d)$ is the contact value of the radial distribution function of the hardsphere reference fluid and is given by ${ }^{47}$

$$
g_{\mathrm{HS}}(d)=\frac{1-0.5 \eta}{(1-\eta)^{3}}
$$

The Mayer function $F_{\mathrm{AB}}$ of the square-well potential energy, of depth $-\epsilon_{\mathrm{w}}$, between two bonding molecules $\mathrm{A}$ and $\mathrm{B}$ is given by

$$
F_{\mathrm{AB}}=\exp \left(\frac{\epsilon_{\mathrm{w}}}{k T}\right)-1
$$

The contribution due to the long-range dispersion forces between like- and unlike-pair molecules is given at the meanfield level ${ }^{49}$

$$
f_{\mathrm{M}}=-\frac{1}{2} \sum_{i} \alpha_{i j} \rho_{i} \rho_{j}
$$

where

$$
\alpha_{i j}=-\int \mathrm{dr} \phi_{i j}(r) \quad(i, j=\mathrm{A}, \mathrm{B})
$$

An isotropic interaction potential of an inverse sixth power law decay is applied,

$$
\phi_{i j}(r)=-4 \epsilon_{i j}\left(\frac{d}{r}\right)^{6} H(r-d) \quad r>d
$$

where $\epsilon_{i j}$ is the energy parameter and $H$ is the Heaviside step function.

\section{Phase Diagram Calculation}

On the basis of the free energy model proposed above, all the thermodynamic properties can be evaluated from eqs 1-11 by using standard thermodynamic relationships. The chemical potential of species $i$ is given by

$$
\mu_{i}=\left(\frac{\partial f_{\mathrm{T}}}{\partial \rho_{i}}\right)_{T, V, \rho_{j \neq i}}
$$

and the equilibrium pressure is thus obtained by

$$
P=\sum_{i} \mu_{i} \rho_{i}-f_{\mathrm{T}}
$$

These functions are necessary to determine the critical lines and phase behavior of the mixture. According to the Gibbs phase rule, there is only one degree of freedom left for a three-phasecoexisting binary system. At a fixed temperature, the criteria for a three-phase equilibrium binary mixture require that the pressure and chemical potential of each component should be the same in all phases. Thus, the equilibrium criteria used to determine the phase equilibrium are shown below ${ }^{31}$

$$
\begin{aligned}
& \mu_{\mathrm{A}}^{\alpha}\left(\rho_{\mathrm{A}}{ }^{\alpha}, \rho_{\mathrm{B}}{ }^{\alpha}, T\right)=\mu_{\mathrm{A}}^{\beta}\left(\rho_{\mathrm{A}}^{\beta}, \rho_{\mathrm{B}}{ }^{\beta}, T\right)=\mu_{\mathrm{A}}^{\gamma}\left(\rho_{\mathrm{A}}{ }^{\gamma}, \rho_{\mathrm{B}}{ }^{\gamma}, T\right) \\
& \mu_{\mathrm{B}}^{\alpha}\left(\rho_{\mathrm{A}}{ }^{\alpha}, \rho_{\mathrm{B}}{ }^{\alpha}, T\right)=\mu_{\mathrm{B}}{ }^{\beta}\left(\rho_{\mathrm{A}}{ }^{\beta}, \rho_{\mathrm{B}}{ }^{\beta}, T\right)=\mu_{\mathrm{B}}{ }^{\gamma}\left(\rho_{\mathrm{A}}{ }^{\gamma}, \rho_{\mathrm{B}}{ }^{\gamma}, T\right)
\end{aligned}
$$




$$
P^{\alpha}\left(\rho_{\mathrm{A}}^{\alpha}, \rho_{\mathrm{B}}{ }^{\alpha}, T\right)=P^{\beta}\left(\rho_{\mathrm{A}}{ }^{\beta}, \rho_{\mathrm{B}}{ }^{\beta}, T\right)=P^{\gamma}\left(\rho_{\mathrm{A}}{ }^{\gamma}, \rho_{\mathrm{B}}^{\gamma}, T\right)
$$

Note that the superscript $\alpha$ represents the lower liquid phase, $\beta$ is the upper liquid phase, and $\gamma$ is the vapor phase. These six equations (14) can be solved simultaneously by numerical methods to obtain the six equilibrium bulk phase compositions, having two densities in each phase.

\section{Density Functional Approach}

The properties of the vapor-liquid and liquid-liquid interfaces between coexisting phases of binary mixtures can be calculated with the application of density functional theory. ${ }^{50}$ Using a local density approximation, the Helmholtz free energy density of a nonuniform binary mixture can be expressed as a functional of the local densities $\rho_{i}(r)^{39-41}$

$$
\begin{aligned}
& F\left(\rho_{i}(r)\right)=\int \mathrm{d} r f_{\mathrm{R}}\left(\rho_{i}(r)\right)+\int \mathrm{d} r f_{\mathrm{A}}\left(\rho_{i}(r)\right)+ \\
& \frac{1}{2} \sum_{i j} \iint \mathrm{d} r \mathrm{~d} r^{\prime} \phi_{i j}\left(\left|r-r^{\prime}\right|\right) \rho_{i}(r) \rho_{j}\left(r^{\prime}\right)
\end{aligned}
$$

where $f_{\mathrm{R}}\left(\rho_{i}(r)\right)$ and $f_{\mathrm{A}}\left(\rho_{i}(r)\right)$ are considered to be functions of local densities $\rho_{i}(r)$ and are given by eqs 2 and 4 .

Consider a planar interface between coexisting phases. The grand potential functional $\Omega\left(\rho_{i}(r)\right)$ for an inhomogeneous binary mixture can be written as follows

$$
\Omega\left(\rho_{i}(r)\right)=F\left(\rho_{i}(r)\right)-\sum_{i} \mu_{i} \int \mathrm{d} r \rho_{i}(r)
$$

The equilibrium density profiles across an interface separating two coexisting phases are obtained through the minimization of the grand potential functional $\Omega\left(\rho_{i}(r)\right) .{ }^{50}$ Setting the derivative of $\Omega\left(\rho_{i}(r)\right)$ with respect to $\rho_{i}(r)$ equal to zero yields a set of coupled integral equations at equilibrium chemical potentials $\mu_{i}$

$$
\mu_{\mathrm{R}}\left(\rho_{i}(r)\right)+\mu_{\mathrm{A}}\left(\rho_{i}(r)\right)=\mu_{i}-\sum_{i j} \int \mathrm{d} r^{\prime} \phi_{i j}\left(\left|r-r^{\prime}\right|\right) \rho_{j}\left(r^{\prime}\right)
$$

where

$$
\begin{aligned}
& \mu_{\mathrm{R}}\left(\rho_{i}(r)\right)=\frac{\partial f_{\mathrm{R}}\left(\rho_{i}(r)\right)}{\partial \rho_{i}(r)} \\
& \mu_{\mathrm{A}}\left(\rho_{i}(r)\right)=\frac{\partial f_{\mathrm{A}}\left(\rho_{i}(r)\right)}{\partial \rho_{i}(r)}
\end{aligned}
$$

The equilibrium bulk densities are evaluated from eq 14 as described in the previous section. These equilibrium densities provide the boundary conditions for the Euler-Lagrange equations for the density profiles, eq 19, which can be numerically solved by an iterative method. ${ }^{35}$ Once the equilibrium density profiles are determined, the interfacial tension $\sigma$ is easily evaluated from

$$
\sigma=\frac{\Omega\left(\rho_{i}(r)\right)+P V}{A}
$$

where $P$ is the equilibrium pressure, $V$ is the volume of the system, and $A$ represents the planar interfacial area between coexisting phases. There are three interfacial tensions, $\sigma_{\alpha \beta}, \sigma_{\alpha \gamma}$, and $\sigma_{\beta \gamma}$, for a three-phase ( $\alpha, \beta$, and $\gamma$ phase) coexisting system. Once these three interfacial tensions are evaluated, the wetting behavior of the $\beta$ phase at the $\alpha-\gamma$ interface can be determined by examining three tensions that obey Neumann's inequality or Antonow's rule. The wetting transition temperature $T_{\mathrm{w}}$ is defined as the temperature at which the relation of interfacial tensions alters from Neumann's inequality to Antonow's rule or vice versa.

\section{Results and Discussion}

All the calculations are performed in reduced units, $T^{*}=$ $k T / \epsilon_{\mathrm{AA}}, \mu_{i}^{*}=\mu_{i} / \epsilon_{\mathrm{AA}}, P^{*}=P d^{3} / \epsilon_{\mathrm{AA}}, \epsilon_{i j} *=\epsilon_{i j} / \epsilon_{\mathrm{AA}}, \epsilon_{\mathrm{w}} *=\epsilon_{\mathrm{W}} /$ $\epsilon_{\mathrm{AA}}, \alpha_{i j} *=3 \alpha_{i j} / 16 \pi d^{3} \epsilon_{\mathrm{AA}}, K_{\mathrm{AB}} *=K_{\mathrm{AB}} / d^{3}$, and $\rho_{i}^{*}=\rho_{i} d^{3}$. It should be noted that $K_{\mathrm{AB}} *=1.0 \times 10^{-5}$ in all our calculations. ${ }^{39-41}$ Both the phase behavior and interfacial wetting behavior of the system are dominated by the interaction energy parameters $\alpha_{\mathrm{AA}}{ }^{*}, \alpha_{\mathrm{AB}}{ }^{*}, \alpha_{\mathrm{BB}}{ }^{*}$, and $\epsilon_{\mathrm{w}}{ }^{*}$. In all our calculations, $\alpha_{\mathrm{AA}} *=1.0$ as a reference energy parameter. In the following, the effect of the other three parameters $\alpha_{\mathrm{AB}} *, \alpha_{\mathrm{BB}} *$, and $\epsilon_{\mathrm{w}} *$ on the phase behavior and the interfacial wetting behavior will be discussed in detail.

6.1. Effect of $\alpha_{A B} *$ on the Phase Behavior and the Interfacial Wetting Behavior $\left(\epsilon_{\mathrm{w}} *=0, \alpha_{\mathrm{AA}} *=1.0\right.$, and $\alpha_{\mathrm{BB}}$ * = 1.2). For comparison, we first present the results of nonassociating, $\epsilon_{\mathrm{w}}{ }^{*}=0$, mixtures. The energy parameters, $\alpha_{\mathrm{AA}}{ }^{*}$ $=1.0$ and $\alpha_{\mathrm{BB}} *=1.2$, are chosen for the model binary mixture, and the phase behavior is dominated by the total interaction strength between unlike-pair molecules $\alpha_{A B} *$. When the total interaction strength between unlike-pair molecules $\alpha_{A B} *$ is weak, say $\alpha_{\mathrm{AB}} *=0.5$, the mutual solubility between $\mathrm{A}$ and $\mathrm{B}$ is small. As one can see in the phase diagram of vapor-liquid-liquid $(\gamma-\beta-\alpha)$ equilibrium in Figure 5a, the mutual solubility between $\mathrm{A}$ and $\mathrm{B}$ remains very small, even when the system is close to its upper critical end point (UCEP). Note that the $x$-axis in Figure 5 is the mole fraction of component $\mathrm{B}, x_{\mathrm{B}}$. The composition of the vapor $\gamma$ phase is dominated by the more volatile compound $\mathrm{A}$. The mixture exhibits vapor-liquid-liquid equilibrium with an UCEP where the liquid $\alpha$ (A-rich) phase and the vapor $\gamma$ phase merge into a single phase. The variation of pressure as a function of temperature is also shown in Figure 5. This mixture belongs to the type III mixture group of van Konynenburg and Scott. ${ }^{28}$

When the total interaction strength $\alpha_{A B} *$ is increased, the mutual solubility between $\mathrm{A}$ and $\mathrm{B}$ is enhanced. Figure $5 \mathrm{~b}$ shows the phase diagram for the mixture of $\alpha_{\mathrm{AB}} *=0.85$. Obviously, the mutual solubility between A and B increases dramatically along with an increase in the temperature. This mixture also exhibits a liquid-liquid miscibility gap coexisting with its vapor $\gamma$ phase with an UCEP where the two liquid phases, $\alpha$ (A-rich) and $\beta$ (B-rich), merge into a single liquid phase. In contrast to the case of $\alpha_{\mathrm{AB}} *=0.5$, this mixture $\left(\alpha_{\mathrm{AB}} *=0.85\right)$ is then classified as a type II mixture of van Konynenburg and Scott. ${ }^{28}$

Note that the variation of the total interaction strength $\alpha_{\mathrm{AB}}{ }^{*}$ would induce a transition of phase behavior from that of type III to that of type II. There exists an UCEP either in the type III mixture or in the type II mixture. The variation of the UCEP as a function of the total interaction strength $\alpha_{\mathrm{AB}} *$ is delineated in Figure 6. For $\alpha_{\mathrm{AB}} *<0.78$, the mixture exhibits the phase behavior of type III. The CEP of $\alpha$ and $\gamma$ phases decreases along with an increase in $\alpha_{A B} *$ reaching a minimum and then increases up to its tricritical point, $\alpha_{\mathrm{AB}} *=0.78$. The tricritical point (TCP) is a critical point at which three phases $\alpha, \beta$, and $\gamma$ merge simultaneously into a single phase. On the other hand, the mixture exhibits the phase behavior of type II for $\alpha_{\mathrm{AB}} *>0.78$. The CEP of $\alpha$ and $\beta$ phases decreases dramatically along with an increase of $\alpha_{\mathrm{AB}} *$. 


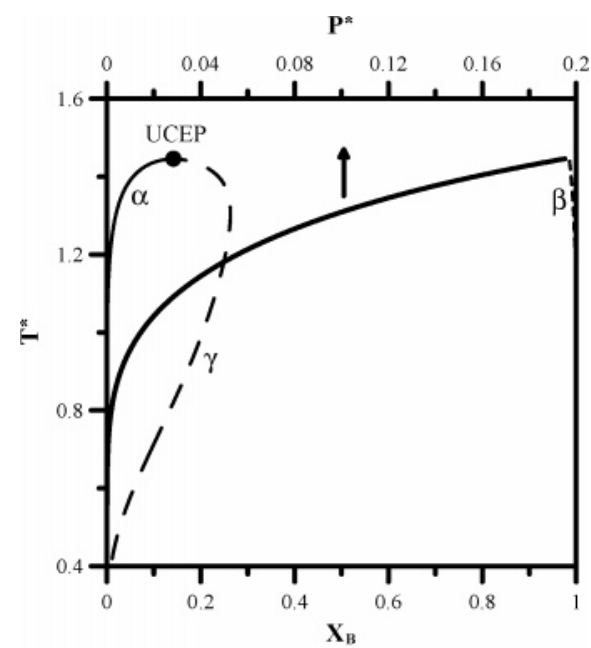

(a)

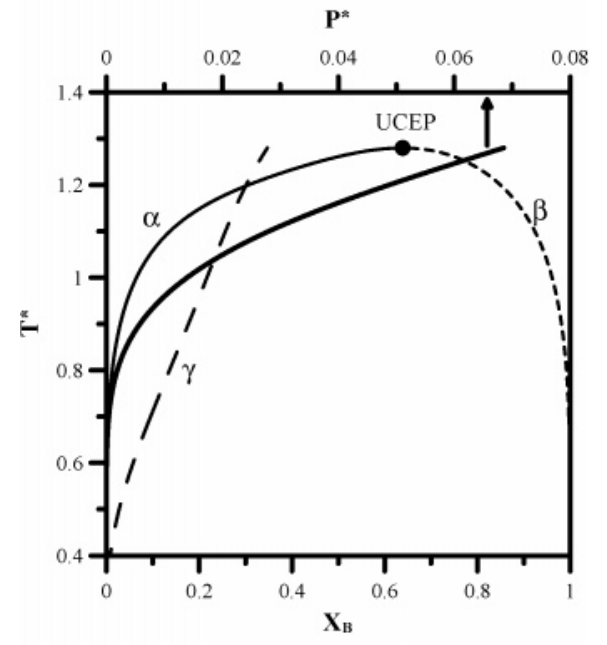

(b)

Figure 5. Vapor-liquid-liquid $(\gamma-\beta-\alpha)$ equilibrium phase diagrams of the binary mixture of equal-sized spheres with $\alpha_{\mathrm{AA}} *=1.0, \alpha_{\mathrm{BB}} *=$ $1.2, \epsilon_{\mathrm{w}}{ }^{*}=0$ and (a) $\alpha_{\mathrm{AB}} *=0.5$ or (b) $\alpha_{\mathrm{AB}} *=0.85$. The temperature vs composition curves are represented as follows: (solid curve) $\alpha$ phase; (dashed curve) $\beta$ phase; and (long dashed curve) $\gamma$ phase. The temperature vs pressure curve is represented as a thick solid curve.

Consider the wetting behavior of the $\beta$ phase at the $\alpha-\gamma$ interface for the system under three-phase coexistence at $\alpha_{\mathrm{AB}}$ * $=0.85$. The system would fall into the region of a type II mixture, as shown in Figure 6. A wetting transition occurs at $T_{\mathrm{W}}=1.148$, while the system is approaching its UCEP $\left(T_{\mathrm{UCEP}}\right.$ $=1.280$ ) from below. When $T^{*} \geq T_{\mathrm{W}}$, the $\beta$ phase dewets the $\alpha-\gamma$ interface. In other words, a small amount of the $\alpha$ phase forms an intruding layer separating the $\beta$ and $\gamma$ phases, as illustrated in Figure 3c*. Besides, this wetting transition between partial wetting and nonwetting is found to be second-order. These results are consistent with a previous study on type II mixtures by Tarazona et al. ${ }^{34}$ In general, the wetting transition temperature as a function of $\alpha_{\mathrm{AB}} *$ is delineated in Figure 6. Note that, when $\alpha_{A B} *<0.6$, the $\beta$ phase almost exhibits partialwetting behavior over the whole three-phase-coexisting temperature region. That is, the wetting transition temperature is too close to the UCEP to be distinguished.

In this study, the order of wetting transitions is also determined by strictly following the method of Tarazona and Evans. ${ }^{34} \mathrm{~A}$ wetting transition is said to be first-order if the temperature dependence of wetting film thickness exhibits a

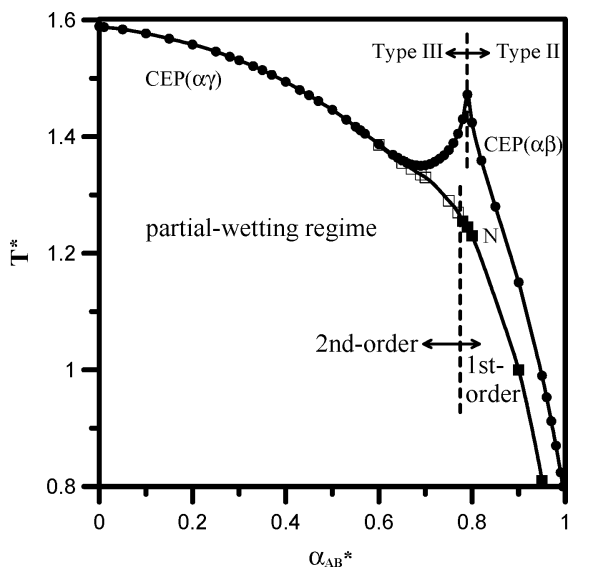

Figure 6. Effect of $\alpha_{\mathrm{AB}} *$ on critical end points (circles) and wetting transition temperatures (diamonds) under the condition of $\epsilon_{\mathrm{w}}{ }^{*}=0$, $\alpha_{\mathrm{AA}} *=1.0$, and $\alpha_{\mathrm{BB}} *=1.2$. Note that there is a transition of phase behavior from that of type III to that of type II mixtures as $\alpha_{A B} *$ increases. The symbol " $\mathrm{N}$ " stands for the regime of the $\beta$ phase exhibiting nonwetting behavior. The open symbols represent the areas where the wetting transition is second-order, and the filled symbols represent those where the wetting transition is first-order.

discontinuity at the wetting transition temperature. On the other hand, if the wetting film thickness grows gradually and diverges at the wetting transition temperature, the wetting transition is identified as second-order. The variation of the order of wetting transitions as a function of $\alpha_{A B} *$ is shown in Figure 6. All the wetting transitions for $\alpha_{A B} *<0.775$ are second-order, and they are first-order for $\alpha_{\mathrm{AB}} *>0.775$, as shown in Figure 6. It is interesting to note that Dietrich and co-workers ${ }^{37,38}$ also provide a complete assessment of the connection between the wetting behavior and the phase behavior, limited to type II and III mixtures. In the former study, binary mixtures consisting of species of different sizes were also analyzed, and the variation of the order of wetting transitions was also scanned over the parameter space..$^{38}$

6.2. Effect of $\epsilon_{\mathrm{w}}{ }^{*}$ on the Phase Behavior and the Interfacial Wetting Behavior $\left(\alpha_{\mathrm{AA}} *=1.0, \alpha_{\mathrm{BB}} *=1.2\right.$, and $\left.\alpha_{\mathrm{AB}} *=0.85\right)$. Now, turn on the effect of the associating interaction between unlike-pair molecules by increasing the strength of $\epsilon_{\mathrm{w}}{ }^{*}$. For small values of $\epsilon_{\mathrm{w}}{ }^{*}$, say $\epsilon_{\mathrm{w}} *<5$, the temperature-composition projection along its triple line is quite insensitive to association at all temperatures. When $\epsilon_{\mathrm{w}} *$ is gradually increased to higher strengths, say $\epsilon_{\mathrm{w}} * \geq 6$, the association effect is enhanced, especially at low temperatures. The association effect can be easily delineated by the number density of bonded molecules $\rho_{\text {bond }}{ }^{*}$, which is defined as $\rho_{\text {bond }} *=\rho_{\mathrm{A}} *\left(1-\chi_{\mathrm{A}}\right)=\rho_{\mathrm{B}} *(1-$ $\left.\chi_{\mathrm{B}}\right)$. Figure 7 shows the temperature dependence of $\rho_{\mathrm{bond}} *$ for the mixtures of $\alpha_{\mathrm{AA}} *=1.0, \alpha_{\mathrm{BB}} *=1.2$, and $\alpha_{\mathrm{AB}} *=0.85$. It is interesting to note that $\rho_{\text {bond }} *$ in the $\alpha$ phase almost coincides with $\rho_{\text {bond }} *$ in the $\beta$ phase, while $\rho_{\text {bond }} *$ in the vapor $\gamma$ phase is too small $\left(<10^{-5}\right)$ to be perceptible. When $\epsilon_{\mathrm{w}} *<5$, over the entire three-phase-coexisting regime, the quantity $\rho_{\text {bond }} *$ remains consistently small. Therefore, the phase behavior remains insensitive to $\epsilon_{\mathrm{w}}{ }^{*}$ for $\epsilon_{\mathrm{w}}{ }^{*}<5$. On the other hand, for large values of $\epsilon_{\mathrm{w}} *$, say $\epsilon_{\mathrm{w}} * \geq 6, \rho_{\text {bond }} *$ monotonically increases as the temperature decreases. Especially, $\rho_{\text {bond }} *$ increases dramatically at low temperatures. This phenomenon is consistent with the temperature dependence of hydrogen-bonding formation. It is much easier to form hydrogen bonds at low temperatures. When the temperature is increased, hydrogen bonds are easily broken due to large thermal fluctuations that make the number of hydrogen bonds decrease. 


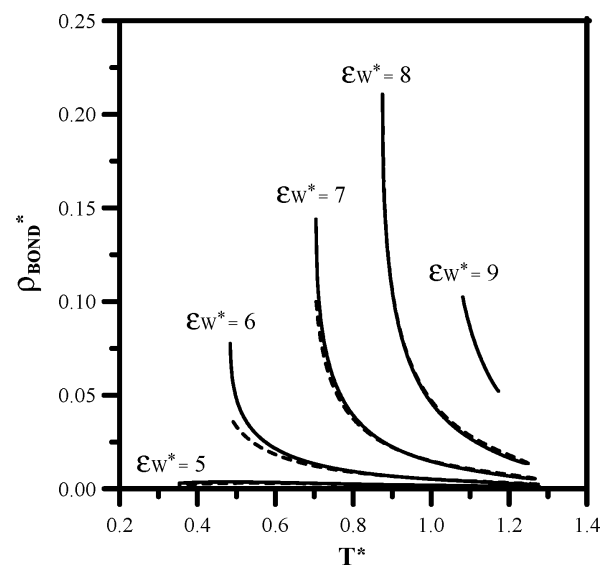

Figure 7. Temperature dependence of the number density of bonded molecules $\rho_{\text {bond }}{ }^{*}$ at five different values of $\epsilon_{\mathrm{w}}{ }^{*}$ for the binary mixture with $\alpha_{\mathrm{AA}} *=1.0, \alpha_{\mathrm{BB}} *=1.2$, and $\alpha_{\mathrm{AB}} *=0.85$.

With an increase in $\epsilon_{\mathrm{w}}{ }^{*}$, the miscibility of compound A and $\mathrm{B}$ increases at low temperatures due to the association effect. Eventually, when $\epsilon_{\mathrm{w}}{ }^{*}=7.85$, a lower critical end point (LCEP), where the two liquid phases $\alpha$ and $\beta$ become identical and coexist with its vapor $\gamma$ phase, emerges to form a closed loop. This association effect induces a transition of phase behavior of the system from that of a type II mixture to that of a type VI mixture, as shown in Figure 8 . The equilibrium compositions of the vapor-liquid-liquid $(\gamma-\beta-\alpha)$ equilibrium phase behavior at two different values of $\epsilon_{\mathrm{w}} *$ are described in Figure 8. The variation of pressure as a function of temperature is also shown in Figure 8 . That is, when $\epsilon_{\mathrm{w}} *<7.85$, the system belongs to the type II mixture group; when $\epsilon_{\mathrm{w}} * \geq 7.85$, the system falls into the category of type VI mixtures, as shown in Figure 9. The closed loop would shrink with further enhancement of the association interaction, as shown in Figure 9. Finally, the closed loop would disappear for $\epsilon_{\mathrm{w}} *>9.03$ and the phase behavior would switch to that of a type I mixture of van Konynenburg and Scott. ${ }^{28}$ That is, a transition of phase behavior from that of type VI to that of type I occurs at $\epsilon_{\mathrm{w}} *=9.03$. In summary, a sequence of phase transitions (type II $\rightarrow$ type VI $\rightarrow$ type I) is observed as the association strength $\epsilon_{\mathrm{w}} *$ increases, as shown in Figure 9.

In the meantime, as the phase behavior of the binary mixture evolves from type II to type VI, another wetting transition temperature emerges accompanying the occurrence of the LCEP. Consider the system with relatively strong association $\epsilon_{\mathrm{w}}{ }^{*}=$ 7.85. While the system temperature approaches either its UCEP from below or its LCEP from above, a wetting transition from a partial-wetting to nonwetting $\beta$ phase occurs. Correspondingly, an upper wetting transition temperature $T_{\mathrm{UW}}=1.078$ and a lower wetting transition temperature $T_{\mathrm{LW}}=0.923$ are found. When $T_{\mathrm{UW}}>T^{*}>T_{\mathrm{LW}}$, the $\beta$ phase exhibits partial-wetting. Beyond these two wetting transition temperatures, the $\beta$ phase completely dewets the $\alpha-\gamma$ interface. In other words, the wetting behavior of the $\beta$ phase at the $\alpha-\gamma$ interface would go through the sequence nonwetting $\rightarrow$ partial-wetting $\rightarrow$ nonwetting along with increasing temperature, as schematically shown in Figure $8 \mathrm{~b}$. This sequential wetting transition is the so-called reentrant wetting. ${ }^{43}$

Figure 9 illustrates the variation of the critical end points and the wetting transition temperatures as a function of $\epsilon_{\mathrm{w}}{ }^{*}$. For type II mixtures, both $T_{\mathrm{UW}}$ and $T_{\mathrm{UCEP}}$ remain almost constant for $\epsilon_{\mathrm{w}}{ }^{*}<5$, as one can see in Figure 9. It should be noted that the lower wetting transition temperature $T_{\mathrm{LW}}$ emerges in the regime of type II mixtures $\left(7.85>\epsilon_{\mathrm{w}}{ }^{*} \geq 7.50\right)$ close to the

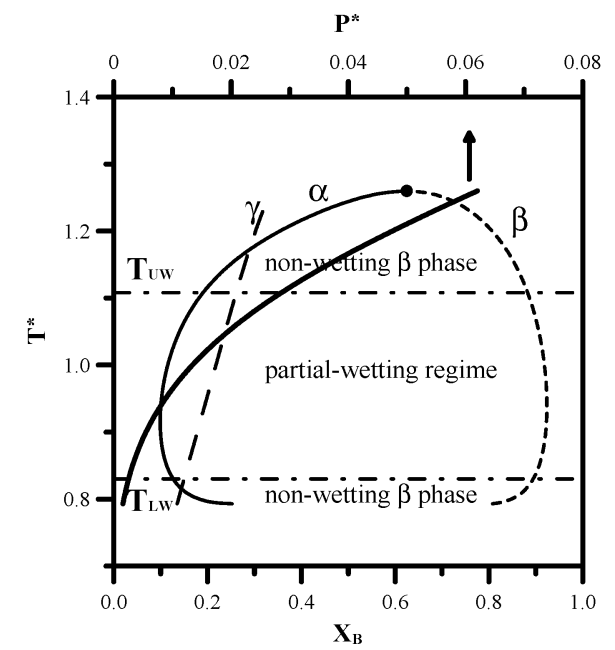

(a)

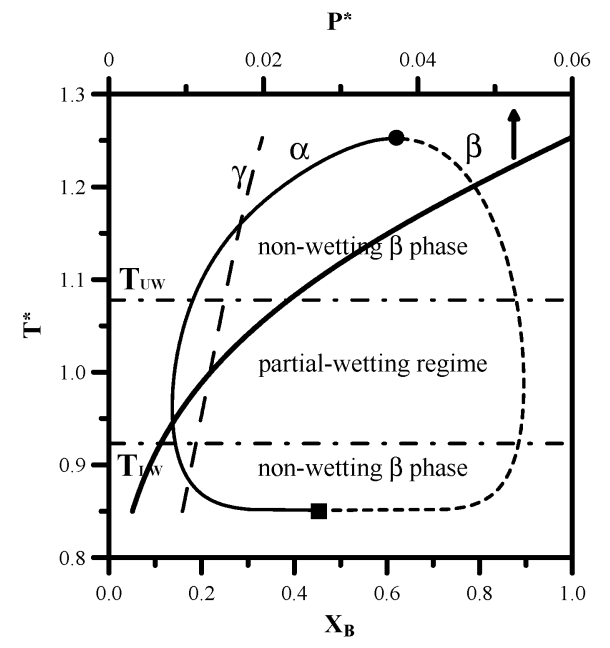

(b)

Figure 8. Vapor-liquid-liquid $(\gamma-\beta-\alpha)$ equilibrium phase diagrams of the binary mixture of equal-sized spheres with $\alpha_{\mathrm{AA}} *=1.0, \alpha_{\mathrm{BB}} *=$ $1.2, \alpha_{\mathrm{AB}} *=0.85$, and (a) $\epsilon_{\mathrm{w}} *=7.50$ or (b) $\epsilon_{\mathrm{w}} *=7.85$. The temperature vs composition curves are represented as follows: (solid curve) $\alpha$ phase; (dashed curve) $\beta$ phase; and (long dashed curve) $\gamma$ phase. The temperature vs pressure curve is represented as a thick solid curve.

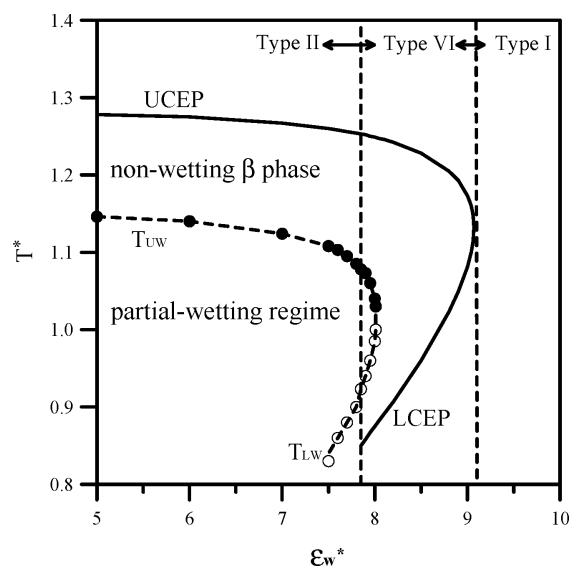

Figure 9. Effect of $\epsilon_{\mathrm{w}}{ }^{*}$ on critical end points and wetting transition temperatures under the condition of $\alpha_{\mathrm{AA}} *=1.0, \alpha_{\mathrm{BB}} *=1.2$, and $\alpha_{\mathrm{AB}} *$ $=0.85$. Note that there is a sequence of phase transitions, type II $\rightarrow$ type VI $\rightarrow$ type I mixtures, as $\epsilon_{\mathrm{w}} *$ increases.

regime of type VI mixtures, as shown in Figure 9. Note that in Figure 9 the lower part of the phase diagram starts to close up 


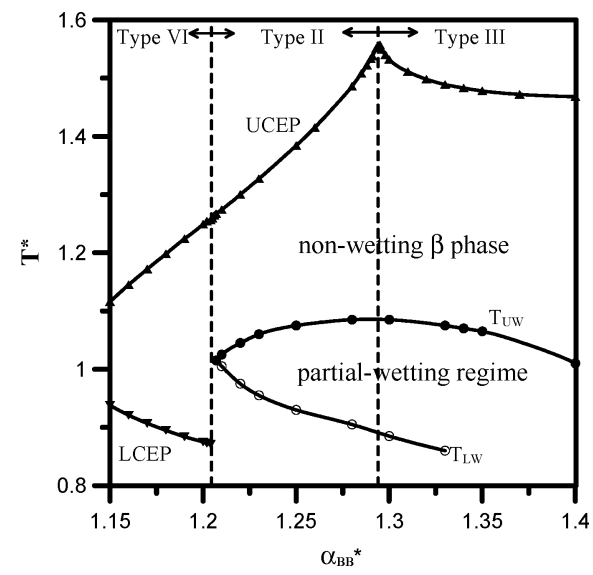

Figure 10. Effect of $\alpha_{\mathrm{BB}}{ }^{*}$ on critical end points and wetting transition temperatures under the condition of $\alpha_{\mathrm{AA}} *=1.0, \epsilon_{\mathrm{w}} *=8.0$, and $\alpha_{\mathrm{AB}} *$ $=0.85$. Note that there is a sequence of phase transitions, type II $\rightarrow$ type $\mathrm{VI} \rightarrow$ type I mixtures, as $\alpha_{\mathrm{BB}} *$ increases.

and, eventually, form a LCEP when $\epsilon_{\mathrm{w}}{ }^{*}$ is approaching 7.85. Although there is no LCEP in the mixtures with $7.85>\epsilon_{\mathrm{w}}{ }^{*} \geq$ 7.50, the lower wetting transition temperature $T_{\mathrm{LW}}$ is found due to the association effect at low temperatures.

For type VI mixtures $\left(\epsilon_{\mathrm{w}} * \geq 7.85\right)$, both $T_{\mathrm{UW}}$ and $T_{\mathrm{LW}}$ are driven further away from their corresponding critical end points with increasing $\epsilon_{\mathrm{w}} *$. Consequently, the regime of the partialwetting $\beta$ phase shrinks when $\epsilon_{\mathrm{w}}{ }^{*}$ increases. Eventually, $T_{\mathrm{UW}}$ and $T_{\mathrm{LW}}$ merge before the closed-loop phase behavior disappears. For very strong associating cases, $\epsilon_{\mathrm{w}} *>8.05$, the $\beta$ phase dewets the $\alpha-\gamma$ interface over the entire three-phase-coexistence regime, as shown in Figure 5b. For $\epsilon_{\mathrm{w}}{ }^{*}>9.03$, the phase behavior of the system falls into the type I mixture group of van Konynenburg and Scott. ${ }^{28}$ There is no vapor-liquid-liquid three-phase-coexisting region for type I mixtures; thus, no wetting behavior can be further explored.

6.3. Effect of $\alpha_{\mathrm{BB}} *$ on the Phase Behavior and the Interfacial Wetting Behavior $\left(\epsilon_{\mathrm{w}}^{*}=8.0, \alpha_{\mathrm{AA}} *=1.0\right.$, and $\left.\alpha_{\mathrm{AB}} *=\mathbf{0 . 8 5}\right)$. Let's fix the strength of the association interaction $\epsilon_{\mathrm{w}}{ }^{*}$ at a relatively strong level, $\epsilon_{\mathrm{w}}{ }^{*}=8.0$, and further examine the effect of the total interaction strength between two $\mathrm{B}$ molecules $\alpha_{\mathrm{BB}} *$ on the phase behavior and the interfacial wetting behavior.

At small values of $\alpha_{\mathrm{BB}} *\left(\alpha_{\mathrm{BB}} *<1.2045\right)$, the system exhibits type VI phase behavior. The variation of the CEP as a function of $\alpha_{\mathrm{BB}} *$ is shown in Figure 10. When the total interaction strength $\alpha_{\mathrm{BB}} *$ increases, the association effect is diminished, especially, at low temperatures. The vapor-liquid-liquid threephase-coexisting regime is widened as $\alpha_{\mathrm{BB}} *$ increases. Furthermore, the LCEP of the $\alpha$ and $\beta$ phases would disappear at large values of $\alpha_{\mathrm{BB}} *\left(\alpha_{\mathrm{BB}} *>1.2045\right)$ and the system would have a transition into type II phase behavior. The UCEP of the $\alpha$ and $\beta$ phases increases along with an increase in $\alpha_{\mathrm{BB}} *$, as shown in Figure 10. If the interaction strength $\alpha_{\mathrm{BB}} *$ is further increased $\left(\alpha_{\mathrm{BB}} *>1.294\right)$, the system would have another transition into type III phase behavior. Note that the UCEP of the $\alpha$ and $\gamma$ phases slightly decreases along with an increase in $\alpha_{\mathrm{BB}} *$ in the regime of type III mixtures.

Consider the wetting behavior of the $\beta$ phase at the $\alpha-\gamma$ interface. The $\beta$ phase exhibits nonwetting behavior over the whole regime of type VI phase behavior, as shown in Figure 10. While in the regime of type II phase behavior, the wetting behavior of the $\beta$ phase at the $\alpha-\gamma$ interface would go through the sequence nonwetting $\rightarrow$ partial-wetting $\rightarrow$ nonwetting along with increasing temperature. Although there is no LCEP in this

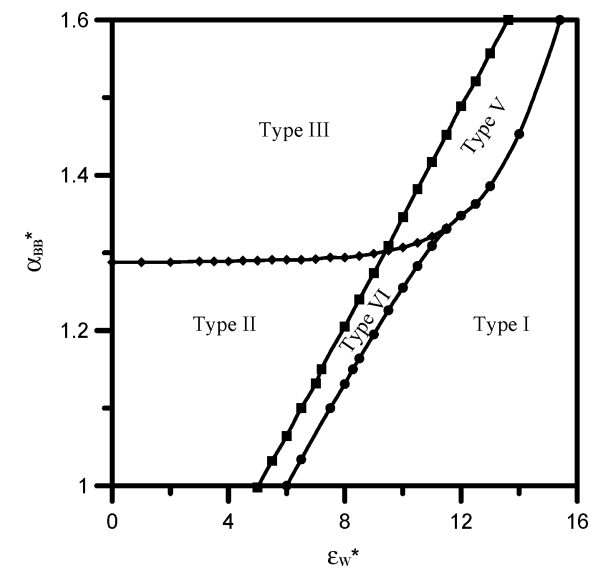

Figure 11. Global phase behavior exhibited by the binary associating mixtures for various $\alpha_{\mathrm{BB}} *$ and $\epsilon_{\mathrm{W}}{ }^{*}$.

regime, the lower wetting transition temperature $T_{\mathrm{LW}}$ appears simply due to the strong association effect at low temperatures. Similarly, in the regime of type III phase behavior $(1.294<$ $\alpha_{\mathrm{BB}} * \leq 1.33$ ), there also exists a reentrant wetting: nonwetting $\rightarrow$ partial-wetting $\rightarrow$ nonwetting for the $\beta$ phase at the $\alpha-\gamma$ interface.

For $\alpha_{\mathrm{BB}} *>1.33$, the lower wetting transition temperature disappears. There exists only the upper wetting transition temperature. That is, for temperatures above $T_{\mathrm{UW}}$, the $\beta$ phase exhibits nonwetting behavior at the $\alpha-\gamma$ interface; for temperatures below $T_{\mathrm{UW}}$, the $\beta$ phase exhibits partial-wetting behavior at the $\alpha-\gamma$ interface.

6.4. Effect of $\alpha_{\mathrm{BB}} *$ and $\epsilon_{\mathrm{w}}{ }^{*}$ on the Global Phase Diagram and the Interfacial Wetting Behavior $\left(\alpha_{\mathrm{AA}} *=1.0\right.$ and $\alpha_{\mathrm{AB}} *$ $=\mathbf{0 . 8 5})$. In the last three sections, we have discussed the effect of the interaction energy parameters $\alpha_{\mathrm{BB}}{ }^{*}, \alpha_{\mathrm{AB}}{ }^{*}$, and $\epsilon_{\mathrm{w}}{ }^{*}$ individually. To get a more general picture, parameters $\alpha_{\mathrm{BB}} *$ and $\epsilon_{\mathrm{w}}{ }^{*}$ are scanned to explore both the phase behavior and the interfacial wetting behavior under the condition of the fixed $\alpha_{\mathrm{AA}} *=1.0$ and $\alpha_{\mathrm{AB}} *=0.85$. Figure 11 shows the global phase behavior of the system as a function of energy parameters $\alpha_{\mathrm{BB}} *$ and $\epsilon_{\mathrm{w}}{ }^{*}$. As mentioned previously, there is a sequence of transitions in phase behavior, type II $\rightarrow$ type VI $\rightarrow$ type I, as $\epsilon_{\mathrm{W}} *$ increases, at a constant $\alpha_{\mathrm{BB}} *$, say $\alpha_{\mathrm{BB}} *=1.2$. When the total strength between $\mathrm{B}$ molecules is increased, say $\alpha_{\mathrm{BB}} *=$ 1.32 , there is another sequence of transitions in phase behavior, type $\mathrm{III} \rightarrow$ type $\mathrm{V} \rightarrow$ type $\mathrm{I}$, along with increasing $\epsilon_{\mathrm{w}}{ }^{*}$, as illustrated in Figure 11. On the other hand, if the association energy parameter $\epsilon_{\mathrm{w}}{ }^{*}$ is fixed at 8.5 , a sequential transition in phase behavior, type I $\rightarrow$ type VI $\rightarrow$ type II $\rightarrow$ type III, is observed as $\alpha_{\mathrm{BB}} *$ increases.

Figure 12 illustrates the interfacial wetting behavior of the $\beta$ phase at the $\alpha-\gamma$ interface, as well as the wetting transition. For small values of $\epsilon_{\mathrm{w}}{ }^{*}\left(\epsilon_{\mathrm{w}} *<7\right)$, the $\beta$ phase undergoes a wetting transition from partial-wetting to nonwetting as temperature increases. For large values of $\epsilon_{\mathrm{w}}{ }^{*}\left(\epsilon_{\mathrm{w}}{ }^{*}>8.2\right)$, the $\beta$ phase always exhibits nonwetting behavior at the $\beta-\gamma$ interface over the whole three-phase-coexisting region. When $8.2>\epsilon_{\mathrm{w}} *$ $>7$, it is possible to observe the reentrant wetting phenomenon, i.e., the wetting behavior of the $\beta$ phase at the $\alpha-\gamma$ interface would go through a sequence of transitions, nonwetting $\rightarrow$ partial-wetting $\rightarrow$ nonwetting, along with increasing temperature. Note that even type II and III mixtures could also exhibit this reentrant wetting phenomenon, as shown in Figure 12. 


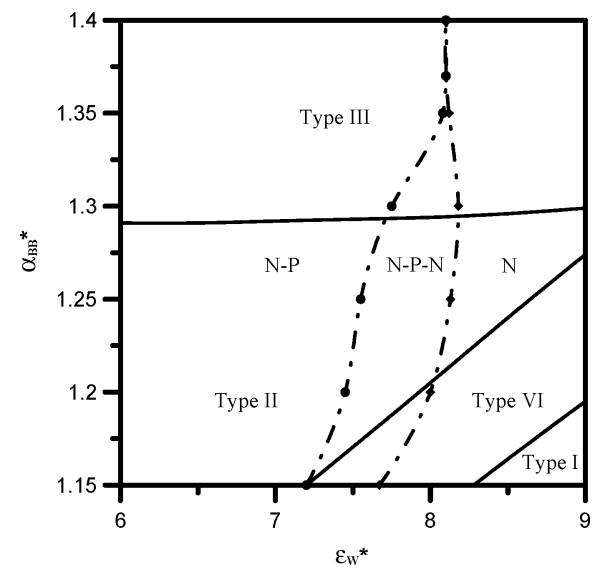

Figure 12. Classification of the interfacial wetting behavior with respect to the binary mixtures for various values of $\alpha_{\mathrm{BB}}{ }^{*}$ and $\epsilon_{\mathrm{w}}{ }^{*}$. The solid curves stand for the boundaries separating regimes of different types of phase behavior, and the dotted-dashed curves separate regimes exhibiting different wetting behaviors. The symbol "P-N" stands for the regime exhibiting a wetting transition of the $\beta$ phase at the $\alpha-\gamma$ interface from partial-wetting to nonwetting as temperature increases to approach its critical point. " $\mathrm{N}$ " stands for the regime of the $\beta$ phase exhibiting only nonwetting behavior over the whole temperature range. "N-P-N" stands for the regime of the $\beta$ phase exhibiting a sequence of wetting transitions, nonwetting $\rightarrow$ partial wetting $\rightarrow$ nonwetting, as temperature increases from LCEP to UCEP.

\section{Conclusions}

A simple Helmholtz free energy model is applied to explore the phase behavior of binary mixtures with associating between unlike-pair molecules. By scanning the associating strength $\epsilon_{\mathrm{w}}{ }^{*}$ and the energy parameter $\alpha_{\mathrm{BB}}{ }^{*}$, the phase behavior of this model mixture is summarized in Figure 11. It is found that this model mixture is able to exhibit five different types, I, II, III, V, and VI, of phase behavior according to the classification scheme of van Konynenburg and Scott. ${ }^{28}$ For interfacial wetting behavior, we restrict our exploration only in all the vapor-liquid-liquid $(\gamma-\beta-\alpha)$ three-phase-coexisting regions of the binary mixtures. The global wetting behavior and wetting transitions are delineated in Figure 12 by scanning the parameter space of the associating strength $\epsilon_{\mathrm{w}}{ }^{*}$ and the energy parameter $\alpha_{\mathrm{BB}} *$. In general, when the associating strength $\epsilon_{\mathrm{w}}{ }^{*}$ is strong, the middle $\beta$ phase exhibits nonwetting behavior at the $\alpha-\gamma$ interface over the whole temperature range of the three-phase-coexisting region. On the other hand, when the associating strength $\epsilon_{\mathrm{w}}{ }^{*}$ is weak, the middle $\beta$ phase exhibits a wetting transition from partial-wetting to nonwetting at the $\alpha-\gamma$ interface along with increasing temperature. While the associating strength $\epsilon_{\mathrm{w}}{ }^{*}$ is intermediate, the middle $\beta$ phase exhibits a sequence of wetting transitions, nonwetting $\rightarrow$ partial-wetting $\rightarrow$ nonwetting, at the interface separating the lower $\alpha$ and upper $\gamma$ phases along with increasing temperature. It would be interesting to relate the theoretical finding in the vapor-liquid-liquid equilibrium binary mixtures in this study to the experimental results. However, all the experimental results in the literature are limited to liquid-liquid equilibrium binary mixtures. Currently, we are in the process of performing the experimental work to determine the wetting behavior as well as wetting transitions in the vaporliquid-liquid equilibrium binary mixtures.
Acknowledgment. This work was supported by the National Science Council of Taiwan, Republic of China.

\section{References and Notes}

(1) Cahn, J. W. J. Chem. Phys. 1977, 66, 3667

(2) Ebner, C.; Saam, W. F. Phys. Rev. Lett. 1977, 38, 1486.

(3) Dietrich, S. In Phase Transitions and Critical Phenomena; Domb,

C., Lebowitz, J. L., Eds.; Academic: London, 1988; Vol. 12, p 1.

(4) Moldover, M. R.; Cahn, J. W. Science 1980, 207, 1073.

(5) Pohl, D. W.; Goldburg, W. I. Phys. Rev. Lett. 1982, 48, 1111.

(6) Schmidt, J. W.; Moldover, M. R. J. Chem. Phys. 1983, 79, 379. 1975 .

(7) Bonn, D.; Hellay, H.; Wegdam, G. H. Phys. Rev. Lett. 1992, 69. 1395.

(8) Kahlweit, M.; Busse, G.; Haase, D.; Jen, J. Phys. Rev. A 1988, 38, 5888 .

(9) Carrillo, E.; Talanquer, V.; Costas, M. J. Phys. Chem. 1996, 100,

(10) Estrada-Alexanders, A.; Garcia-Valenzuela, A.; Guzman, F. J. Phys. Chem. 1991, 95, 219.

(11) Aratono, M.; Kahlweit, M. J. Chem. Phys. 1991, 95, 8578.

(12) Chen, L.-J.; Jeng, J.-F.; Robert, M.; Shukla, K. P. Phys. Rev. A 1990, 42, 4716 .

(13) Smith, D. H.; Covatch, G. L. J. Chem. Phys. 1990, 93, 6870.

(14) Chen, L.-J.; Yan, W.-J. J. Chem. Phys. 1993, 98, 4830.

(15) Chen, L.-J.; Yan, W.-J.; Hsu, M.-C.; Tyan, D.-L. J. Phys. Chem. 1994, 98, 1910

(16) Chen, L.-J.; Lin, S.-Y.; Xyu, J.-W. J. Chem. Phys. 1996, 104, 225.

(17) Kahlweit, M.; Strey, R.; Busse, G. Phys. Rev. E 1993, 47, 4197.

(18) Chen, L.-J.; Hsu, M.-C. J. Chem. Phys. 1992, 97, 690.

(19) Chen, L.-J.; Hsu, M.-C.; Lin, S.-T.; Yang, S.-Y. J. Phys. Chem. 1995, 99, 4687.

(20) Kahlweit, M.; Busse, G. J. Phys. Chem. B 2000, 104, 4939.

(21) Chen, L.-J.; Chiu, C.-D.; Shau, F.-S.; Cheng, W.-J.; Wu, J.-G. J. Phys. Chem. B 2002, 106, 12782.

(22) Yeh, M.-C.; Lin, P.-C.; Chen, L.-J. J. Phys. Chem. B 2004, 108, 9955.

(23) Wu, C.-K.; Chen, L.-J. J. Chem. Phys. 2005, 123 (8), 084506.

(24) Wu, C.-K.; Chen, L.-J. Langmuir 2005, 21, 6883.

(25) Ragil, K.; Meunier, J.; Broseta, D.; Indekeu, J. O.; Bonn, D. Phys Rev. Lett. 1996, 77, 1532.

(26) Ross, D.; Bonn, D.; Meunier, J. Nature 1999, 400, 737.

(27) Bertrand, E.; Dobbs, H.; Broseta, D.; Indekeu, J. O.; Bonn, D.; Meunier, J. Phys. Rev. Lett. 2000, 85, 1282.

(28) van Konynenburg, P. H.; Scott, R. L. Philos. Trans. R. Soc. London, Ser. A 1980, $298,495$.

(29) Gompper, G.; Schick, M. Phase Transitions and Critical Phenomena; Domb, C., Lebowitz, J. L., Eds.; Academic: New York, 1994; Vol. 16 .

(30) Telo da Gama, M. M.; Evans, R. Mol. Phys. 1980, 41, 1091

(31) Telo da Gama, M. M.; Evans, R. Mol. Phys. 1983, 48, 229.

(32) Telo da Gama, M. M.; Evans, R. Mol. Phys. 1983, 48, 251.

(33) Telo da Gama, M. M.; Evans, R. Mol. Phys. 1983, 48, 687.

(34) Tarazona, P.; Evans, R. Mol. Phys. 1983, 48, 799.

(35) Tarazona, P.; Telo da Gama, M. M.; Evans, R. Mol. Phys. 1983 $49,283$.

(36) Tarazona, P.; Evans, R. Mol. Phys. 1984, 52, 847.

(37) Dietrich, S.; Latz, A. Phys. Rev. B 1989, 40, 9204.

(38) Getta, T.; Dietrich, S. Phys. Rev. E 1993, 47, 1856

(39) Yeh, M.-C.; Chen, L.-J. J. Chem. Phys. 2003, $118,8331$.

(40) Talanquer, V.; Oxtoby, D. W. Faraday Discuss. 1999, 112, 91.

(41) Yeh, M.-C.; Chen, C.-M.; Chen, L.-J. J. Phys. Chem. B 2004, 108, 7271 .

(42) Jackson, G. Mol. Phys. 1991, 72, 1365.

(43) Pérez, C.; Roquero, P.; Talanquer, V. J. Chem. Phys. 1994, 100, 5913.

(44) Antonow, G. N. J. Chem. Phys. 1907, 5, 372.

(45) Buff, F. P. Encyclopedia of Physics; Flugge, S., Ed.; Springer:

Berlin, 1960; Vol. 10, Section 7, pp 298 and 299.

(46) Widom, B. J. Chem. Phys. 1975, 62, 1332.

(47) Carnaham, N. F.; Starling, K. E. J. Chem. Phys. 1969, 51, 635.

(48) Green, D. G.; Jackson, G. J. Chem. Phys. 1992, 97, 8672.

(49) Rowlinson, J. S.; Swinton, F. L. Liquid and Liquids mixtures, 3rd ed.; Butterworth: London, 1982.

(50) Evans, R. Adv. Phys. 1979, 28, 143. 